\title{
Classification of carbon materials for developing structure- properties relationships based on the aggregate state of the precursors
}

\author{
Oleksiy V. Khavryuchenko ${ }^{a, *}$, Volodymyr D. Khavryuchenko ${ }^{b}$ \\ ${ }^{a}$ Chemistry Department, Taras Shevchenko National University of Kyiv, Volodymyrska str. 64, Kyiv 01601, Ukraine \\ b CompChem Group, Revutskogo str. 13, Kyiv 02091, Ukraine
}

\begin{abstract}
Modern carbon science lacks an efficient structure-related classification of materials. We present an approach based on dividing carbon materials by the aggregate state of the precursor. The common features in the structure of carbon particles that allow putting them into a group are discussed, with particular attention to the potential energy stored in the carbon structure from different rates of relaxation during the synthesis and prearrangement of structural motifs due to the effect of the precursor structure. Keywords: Carbon material; Classification; Carbonization Received 30 March 2014. Published 20 June 2014.
\end{abstract}

(C) 2014, Dalian Institute of Chemical Physics, Chinese Academy of Sciences. Published by Elsevier B.V. All rights reserved.

\section{Introduction}

The chemistry of carbon materials (CMs) seems terribly tangled for those who try to enter this domain for the first time. Moreover, this feeling can remain after several years of work. This is not surprising since there are almost one thousand types of pure carbon materials known at this moment, and there are even more types of modified and doped ones. Actually this field of science suffers from the lack of classification more than any other areas of materials chemistry.

Although works on the classification of carbon materials have been reported, however, our point of view is that they still do not cover all varieties of CMs and do not give the expected scientific insight into structure-properties relationships. Therefore, in this essay we present a short review on the current state-of-art in the classification of CMs and our suggestions for its improvement. We hope to start a wide discussion in the carbon community.

\section{History}

The first attempts to describe the structure of carbon were by Warren in 1936 [1,2] based on pioneering X-ray diffraction (XRD) experiments on non-crystalline carbon. The concept of "turbostratic" (meaning in-plane rotation of graphene sheets in graphite) carbon was introduced to explain the widening of peaks in XRD patterns. The next step was made by Rosalind Franklin in 1951 [3] when she explained the difference between graphitizing and non-graphitizing carbons (transformed or not into graphite on heating below $2000 \mathrm{~K}$ with no additional treatment, respectively) by the pre-orientation of graphite micro-crystallites in the bulk, which allowed or did not allow further rearrangement.

The works of Rosalind Franklin and co-workers formed the first classification of carbon materials by their relation to graphite as the most thermodynamically stable form. All materials were divided into graphitic (consisting solely of graphite particles) and non-graphitic. The latter materials were subdivided into graphitizing and non-graphitizing, as described above. This classification was purely phenomenological, but still valid. In the 1990s, it was modified to account for the curved carbon planes discovery, which formed a wide range of materials like fullerenes, carbon nanotubes (CNTs) and their derivatives. It was recognized that the crystallites are not necessarily planar [4,5], but the main idea of CMs as structurally reducible to graphene or graphene-derived building blocks remained.

However, with the passage of time, many differences in the properties of CMs have appeared that cannot be attributed to the orientation of the graphitic (even when formed by curved sheets) crystallites in the particle. It became obvious that some general rules besides the tendency to graphitize on heating should be proposed.

\footnotetext{
* Corresponding author. Tel/Fax: +38-044-2354371; E-mail: alexkhavr@gmail.com DOI: 10.1016/S1872-2067(14)60112-1 | http://www.sciencedirect.com/science/journal/18722067 | Chin. J. Catal., Vol. 35, No. 6, June 2014
} 


\section{Common problems}

There are a series of problems that anyone making a classification of CMs will encounter. We list the most important of these.

1. One of the most popular trends in treating CMs is the reduction of their structure to graphene forms and their properties to the properties of edge groups or the graphene basal plane [6,7]. The cross-links between crystallites are usually neglected in their impact on the chemical and physical properties and they are treated simply as mechanical junctions. This shortcoming has a mainly historical origin since CMs were considered as "more or less graphite" for more than a century. Also, the simplified picture of "carbon as graphite" is much easier to comprehend, so many people are naturally inclined to take it as self-evident.

2. Sometimes people tend to reduce the chemistry of CMs to organic chemistry, which neglects their important properties due to their solid state chemistry and porosity. This happens quite often in the interpretation of the acid-base properties of CMs or the surface functionalization of CMs.

3. One should emphasize that "pure carbon" materials in the majority of cases contain not only carbon atoms but also some hydrogen atoms, and although their mass content is not very high, their atomic ratio may reach 30 atom\%. The role of hydrogen in carbon materials is often wrongly underestimated, as it both affects the physical properties (especially magnetism-related ones) of the material and can be used for characterization (e.g. by ${ }^{1} \mathrm{H}$ NMR or echo EPR spectroscopy).

4. The terms used in CMs chemistry are ambiguous. This group of problems can be subdivided into two groups: those related to terms of amorphism and those related to the notation of the types and features of the CMs. For the former, the situation in the classification of amorphous matter and related phenomena is not acceptable. The approach to amorphous solids is still dominated by the concept of an overcooled liquid, which is valid for glasses but not for other types of amorphous materials (including CMs), and there is muddled use of terms like "amorphous", "disordered", "nano-crystalline", etc. The terms used in carbon science are also often not clearly defined (e.g. the term "carbonization" is also used as "pre-carbonization" depending on the area of application). The denotations of
CMs exist as an overlay of traditional names and trademarks, which are different for different manufacturers and research groups. One can look up a typical classification of CMs elsewhere [8] and observe that the origin of the CMs, their structure and some key properties are mixed together in the presenting of the classes of CMs. A typical approach in this case is to define the three "classic" allotropes of carbon (diamond, graphite, and carbyne), derive some of the CMs as defective diamond or defective graphite and put the rest of the materials into the general group of "nanocarbons" and their assemblies.

Several phenomenological characteristics of CMs are used as the basis of a universal classification, namely, porosity, acidity in water, linear edge density, etc. [9]. A number of classifications are developed for some particular types of CMs, e.g. carbon fibers, graphites (implying all graphite-like materials), coals, etc., but most of these cannot be extended beyond their specific area. These classification schemes are very useful in technological applications, but in the majority of cases, they are not suitable for research. A very important step in organizing the terminology in carbon science was performed recently by the editorial board of Carbon journal [10]. However, their systematic organization concerns graphene-related terms only, which is not enough for clarity in terms usage.

So, unfortunately, there is only one structure-related classification of CMs - that derived from Franklin's idea of an ordering as non-graphitizable carbon. Meanwhile, other classifications involve only some particular types of CMs or are phenomenological approached.

\section{Classification}

Bearing in mind all of the above points, we would like to stress another approach in the classification of CMs, namely, by the origin of the precursor. One can note that the properties of a majority of CMs are history-dependent, with the exception of some rare cases, e.g., extremely graphitized (HOPG) samples (some characteristic examples are given in Fig. 1). Even materials with a seemingly ordered structure like CNTs are known to differ from batch to batch significantly. Differences in properties are often attributed to the presence of admixtures. However, unlike the chemistry of molecular compounds, in materials chemistry, this excuse has no sense since any component in
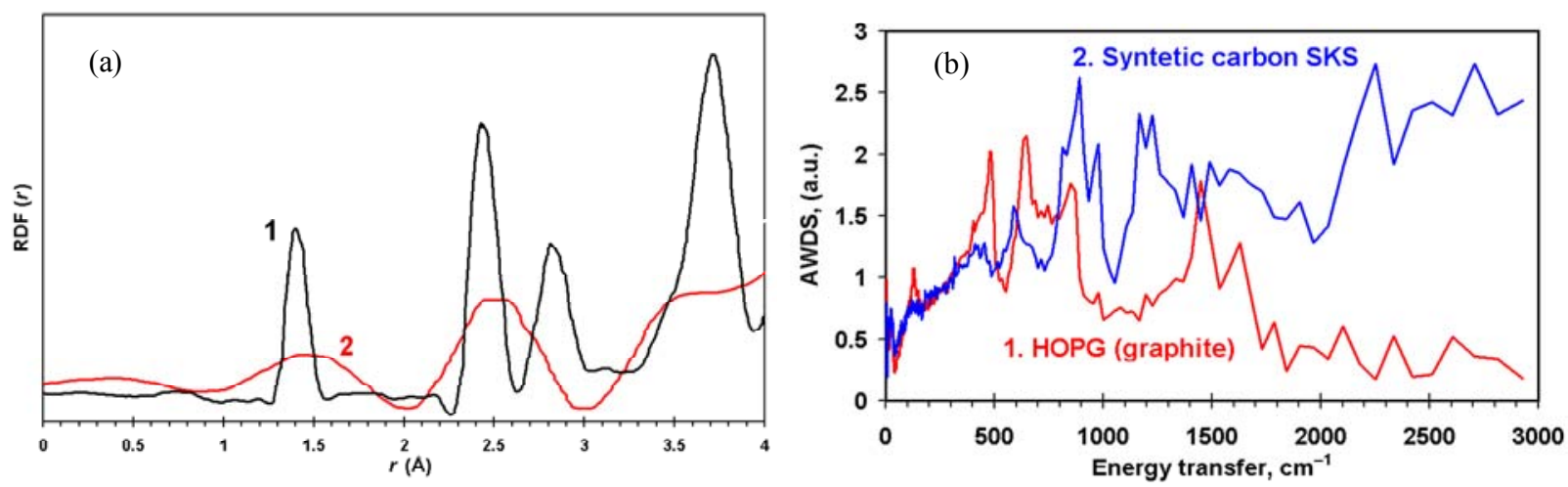

Fig. 1. Examples of structural differences between graphite and non-graphitic CMs. (a) Radial distribution function of graphite (1) [11] and carbon black (2) [12]; (b) Inelastic neutron scattering spectra of HOPG and SKS polymer-derived carbon [13]. 
the mixture which affects the properties cannot be neglected. Most CMs are far from their thermodynamically stable form, so the origin of the precursor would affect the properties of the CM both locally (due to the presence of specific functional groups or the nature of the non-graphene carbon backbone) and in a supramolecular way (by the introduction of domain arrangements in the bulk). The potential energy conserved in carbon particles in a non-ordered (often strained) structure and the presence of metastable groups can affect the chemical properties of the CM by destabilizing the initial state of the reactants and, thus, decreasing the activation barrier for some reactions (e.g. surface modification or interaction with polymers upon filling). Formally, this conserved energy can be measured directly by differential scanning calorimetry, but this macroscopic characteristic of the CM cannot be used solely to differentiate between types of CMs.

On the other hand, some common features are found between different types of CMs produced from the same aggregate state of the precursor. Starting from this, we would like to propose a new approach in classification of CMs. The three big groups of CMs would be, naturally, those that were produced from the gas, liquid, and solid phases (Table 1). Let us characterize them briefly.

\subsection{CMs produced from the gas phase}

The following types of CMs are in this group: carbon black (carbon soot) [14], fullerenes [15], CNTs [16], carbon films grown by epitaxy or vapour deposition (CVD, LVD, etc.) [17], tetrahedral amorphous carbon [18], etc. (one should note that here as well as for the following groups the types of CMs often overlap).

This group can also be subdivided into two groups: carbon particles completely formed in the gas phase (e.g. fullerenes, carbon black, "nanodiamonds" [19]) and those where carbon-rich molecules or proto-particles in the gas phase are attached to a carbon-rich surface (e.g. ta-carbon films, CNTs).

The common feature that all CMs in this group share is that the carbon particles are formed from relatively small excited carbon-rich molecules. As a consequence, the overall process usually takes place in the kinetic controlled regime, resulting in cross-linked dense structures (except for the cases where the particle growth occurs on the surface of a catalyst). The transport of reacting molecules and protoparticles is not sterically hindered, however, the rate of particle growth is limited by the probability of collisions in the gas phase or at the solid-gas interface. Each precursor molecule or protoparticle is potentially a nucleating center (again, except for those materials growing on a catalyst), hence, the growth of the particle can be, to some extent, considered as a coalescence of highly energetic nuclei. Energy dissipation is often an issue for these processes, so local motifs formed as a result of the elementary reactions cannot relax to energetically preferred configurations. The local atomic arrangement (often tetrahedral carbon atoms), thus, does not get to the thermodynamic minima, and potential energy is conserved in the structure mainly in the form of local stresses. These materials are often referred to as "truly amorphous".

As one can observed, this group of CMs is extremely diverse in their chemical and physical properties. Materials grown on a catalyst, e.g. CNTs (Fig. 2(a)), are often very structured and formally can be considered as derivatives of graphene (including the fact that their potential energy is close to that of graphite, so their chemical reactivity is quite low). On the other hand, there is a specific subgroup of CMs formed in high vacuum (e.g. in interstellar space, Fig. 2(b)), which is characterized by a thread-like structure of the allene type and, thus, low chemical reactivity (a low energy of the reacting species and a diluted media are the prerequisites for the formation of such materials) [20].

\subsection{CMs produced from the liquid phase}

This group of CMs comprises materials mainly prepared by hydrothermal (and in a more general case, solvothermal, which include ionic liquids as solvent) synthesis and it is growing very

Table 1

Features of carbon materials obtained from different aggregate states of the precursor.

\begin{tabular}{|c|c|c|c|c|}
\hline \multirow{2}{*}{ Features } & \multicolumn{2}{|c|}{ Gas phase } & \multirow{2}{*}{ Liquid phase } & \multirow{2}{*}{ Solid state } \\
\hline & Formed in gas phase & Formed on support & & \\
\hline Nucleation & Fast, spontaneous & Fast & Slow & Slow \\
\hline Particle growth & Slow & Slow & Moderate & Slow \\
\hline $\begin{array}{l}\text { Types of growth } \\
\text { reactions }\end{array}$ & $\begin{array}{l}\text { Radicals coupling, } \\
\text { radical polymerization }\end{array}$ & $\begin{array}{l}\text { Radicals coupling, } \\
\text { radical polymerization }\end{array}$ & $\begin{array}{l}\text { Acid/base reactions, } \\
\text { cycloaddition }\end{array}$ & $\begin{array}{l}\text { Radicals coupling, rearrangements } \\
\text { in polycyclic hydrocarbons }\end{array}$ \\
\hline Coalescence & High & With the support & Low & Extremely low \\
\hline Mass transfer & Slow & Slow & Moderate & Extremely low \\
\hline Heat dissipation & Hindered & Moderate & High & Moderate \\
\hline Relaxation & Hindered & Hindered & High & Hindered \\
\hline Potential energy & High & High & Low & High \\
\hline Local structure & Tetrahedral C & Tetrahedral C & $\begin{array}{l}\text { Polycyclic hydrocarbons, } \\
\text { residual groups }\end{array}$ & $\begin{array}{l}\text { Polycyclic hydrocarbons, short } \\
\text { unsaturated carbon chains }\end{array}$ \\
\hline Ordering & No ordering & No ordering & Medium-range & $\begin{array}{l}\text { Two-domain structure (graphitized } \\
\text { particles connected by chains) }\end{array}$ \\
\hline $\begin{array}{l}\text { Possible subgroups } \\
\text { (with divergent properties) }\end{array}$ & Formed in high vacuum & Grown on a catalyst & $\begin{array}{l}\text { (1) obtained from monomers; } \\
\text { (2) obtained from soluble } \\
\text { polymers }\end{array}$ & \\
\hline
\end{tabular}


rapidly in the past few years [21]. The common feature of these CMs is that classical acid-base processes take place in their formation, so the structure of the final material can be derived from the precursors' structure by following the network of rather well explored ionic polymerization-depolymerization and functionalization reactions. This group falls into two subgroups: (1) CMs obtained by the polymerization of organic molecules and (2) those derived from soluble polymers upon partial destruction and carbonization. Both types undergo further partial carbonization under critical conditions (high pressure and temperature), which are, however, milder than those used in the two other groups. It should be emphasized that the term "carbonization" in this case has an essentially different chemical sense from that in the third group (solid state carbonization), and which is closer to "precarbonization". The limiting stage of the carbon particle formation is nucleation $[22,23]$, which occurs through the dehydration of carbohydrates or some other low temperature process of $\mathrm{C}=\mathrm{C}$ bond formation with a subsequent cycloaddition to form small fused aromatic moieties (Fig. 2(c)). The transport of molecular and oligomeric precursors to the nuclei is easy due to their high concentration and mobility in solution, so the carbon particles usually grow as spherically-shaped with a low chance of coalescence.

In the majority of cases, the CMs of this group have a dual nature. On one hand, the parts of the material accessed by the solvent are relaxed and contain conventional organic functional groups. On the other hand, the backbone of these CMs can con-
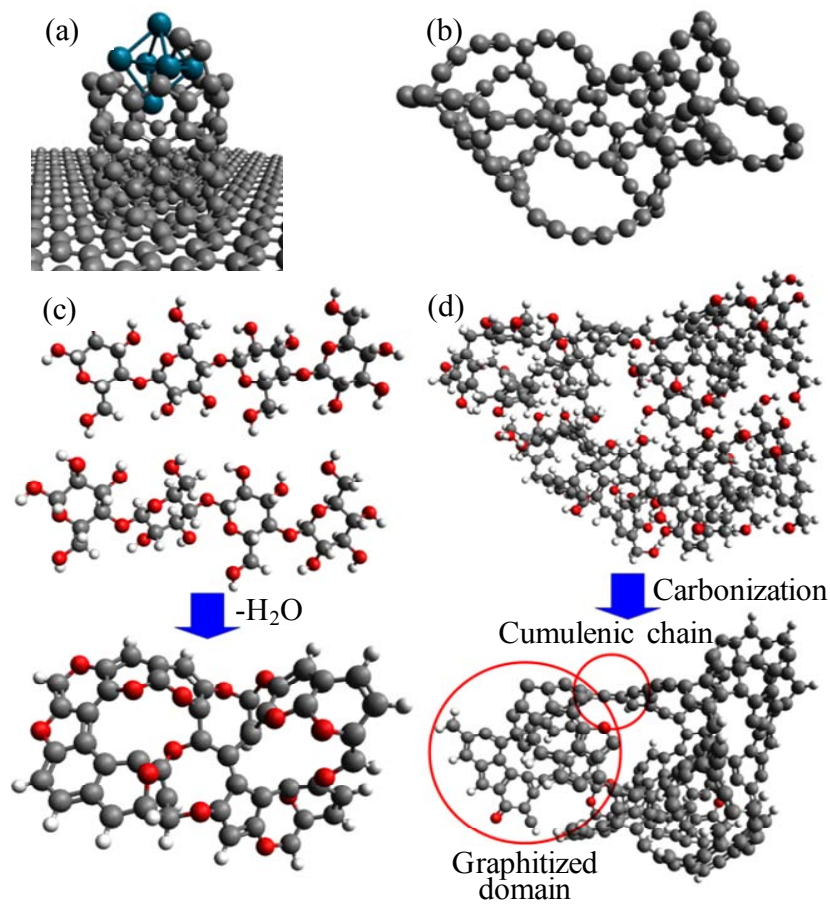

Fig. 2. Models of CMs of different origins. (a) CNTs growing on a metal catalyst; (b) Carbon clusters formed in high vacuum; (c) Formation of carbonaceous material from glucose oligomerization through dehydration with subsequent cycloaddition in water; (d) Formation of amorphous carbon cluster through carbonization of a phenol-formaldehyde resin [24]. tain residual admixture molecules and ions (including structural water molecules), and in general the core of the carbon particles have internal stresses due to non-relaxed conformations of polymeric chains formed during particle growth.

One should emphasize that natural coal and related materials (kerogen, peat, etc.) fall into this group, too. Clearly, the time of their formation was much longer and, thus, the rate of the processes was much slower, which affects the structure and properties of coal significantly.

\subsection{CMs produced from the solid phase}

In most cases, the CMs of this group are produced by the carbonization of polymers $[25,26]$. In the case of viscous liquids (e.g. pitch or polyaromatic hydrocarbons), a radical polymerization occurs first, leading to a polymer, which then undergoes the same processes as a plain polymer. Therefore, the structure of these materials can be considered as post-polymeric. This implies that the CMs of this group often retain partially dehydrogenated/defunctionalized polymeric chains (usually short), which are highly disordered (Fig. 2(d)). More organized carbon subparticles (usually polycyclic hydrocarbons, and not necessarily built up from aromatic hexagons) are kinetic controlled products of these partially dehydrogenated/defunctionalized polymeric chains that undergo condensation under highly critical conditions due to the internal stresses present during carbonization.

The carbonization itself occurs in two distinctive stages. The first, usually called "pre-carbonization" (resembling the "carbonization" in the second group of CMs) involves the elimination of most of the functional groups in the form of volatile molecules, often of organic origin (i.e. tar), and the second can be considered as carbonization in the pure sense. Because of extremely limited mass transport, "nucleation" of carbonized domains dominates over equilibrium growth, while the coalescence of the protoparticles is impossible due to their negligible mobility. Since the chemical processes during the carbonization of polymers occur in extremely confined conditions under a high temperature, the initial disorder of the polymer chains can be "conserved" in the final product and even intensified due to the low possibility for relaxation. However, in contrast to the CMs of the first group (gas-phase derived), these materials are not dense. The short polymeric and post-polymeric chains often have rotational degrees of freedom, with a relatively low content of tetrahedral carbon atoms.

Obviously, this classification is not universal. It involves only neat CMs obtained from non-carbon precursors. A variety of "second-order" materials, such as graphene oxide, exfoliated graphite, synthetic diamonds, etc., produced from some kind of already formed CM, need to be described separately. Several subgroups of the gas-phase formed CMs, namely, (1) grown on a catalyst and (2) formed in high vacuum, can be divided into separate groups. One should keep in mind that the classification by the aggregate state of the precursor is not absolute and does not preclude other types of classification. It is complementary rather than a substitute and, most probably, it can be substantially refined. 


\section{Graphical Abstract}

Chin. J. Catal., 2014, 35: 778-782 doi: 10.1016/S1872-2067(14)60112-1

Classification of carbon materials for developing structure-properties relationships based on the aggregate state of the precursors

Oleksiy V. Khavryuchenko*, Volodymyr D. Khavryuchenko

Taras Shevchenko National University of Kyiv, Ukraine; CompChem Group, Ukraine

Carbon materials can be classified into three groups by the aggregate state of the precursor material: those produced from gas, liquid or solid phase. Characteristic features are often shared by the materials of the same group.

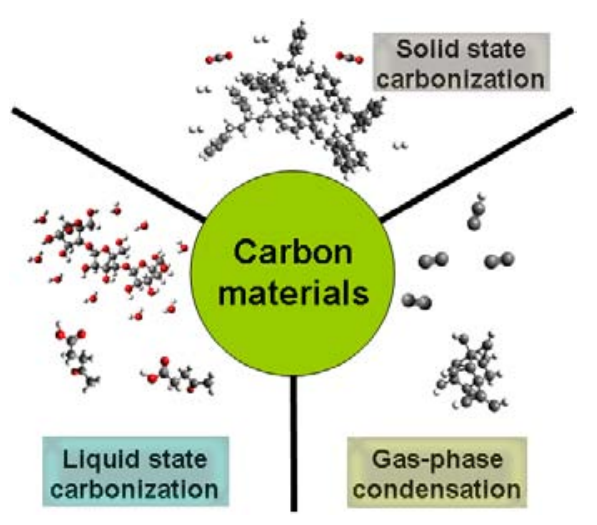

\section{Conclusions}

We proposed a classification of CMs by the aggregate state of the precursor. There are two main features that the CMs in each group share, namely, the rate of relaxation during synthesis and prearrangement of the structural motifs in the carbon particle due to the macro- and microstructure of the precursor material. In the case of gas phase-derived materials, the rate of relaxation is much slower than that of particle growth (due to high temperature or shock wave compression [19]). In the solid state-derived ones, relaxation is hindered by internal stresses. In the liquid phase, relaxation is facilitated at least near the surface. Therefore, a different amount of potential energy is conserved in the carbon particles of the different classes (it is very important to note that the chemistry of the CMs is not reduced to the chemistry of the surface functional groups, so the potential energy of the particle would play a crucial role in its reactivity). Also, carbonized particle nucleation-growth rates are very different for these groups of CMs, which affect the structure of the materials significantly. Prearrangement of the domains in the carbon particles due to the structure of the precursor has an impact both on its local properties (e.g. due to the conservation of non-conventional functional groups) and macroscopic properties, such as graphitization ability and bulk density.

\section{Acknowledgments}

The INS spectra were measured by the author using inverted geometry time-of-flight spectrometers (NERA) installed in an IBR-2 nuclear pulse reactor at the Joint Institute for Nuclear Research (JINR) Dubna, Russia, in the group of Dr. Ireneusz Natkaniec.

\section{References}

[1] Warren B E. JChem Phys, 1934, 2: 551
[2] Warren B E. Phys Rev, 1941, 59: 693

[3] Franklin R E. Proc R Soc Lond Ser A, 1951, 209: 196

[4] Harris P J F. Crit Rev Solid State Mater Sci, 2005, 30: 235

[5] Harris P J F. Phil Mag, 2004, 84: 3159

[6] Marsh H, Rodríguez-Reinoso F. Activated Carbon. London: Elsevier, 2006. 536

[7] László K, Josepovits K, Tombácz E. Anal Sci, 2001, 17(suppl.): i1741

[8] Krueger A. Carbon Materials and Nanotechnology. Weinheim: WILEY-VCH Verlag GmbH \& Co. KGaA, 2010. 475

[9] Stoner R B, Glass J T. Diam Rel Mater, 2012, 23: 130

[10] Bianco A, Cheng H M, Enoki T, Gogotsi Y, Hurt R H, Koratkar N, Kyotani T, Monthioux M, Park C R, Tascon J M D, Zhang J. Carbon, 2013, 65: 1

[11] Fukunaga T, Itoh K, Orimo S, Aoki M, Fujii H. J Alloy Compd, 2001, 327: 224

[12] Darmstadt H, Roy C, Kaliaguinea S, Xu G Y, Auger M, Tuel A, Ramaswamy V. Carbon, 2000, 38: 1279

[13] Khavryuchenko V D, Tarasenko Y A, Khavryuchenko O V, Shkilnyy A I, Lisnyak V V, Stratiichuk D A. Int J Modern Phys B, 2010, 24: 1449

[14] Donnet J B, Johnson M P, Norman D T, Wang T K. Carbon, 2000, 38 : 1885

[15] Darwish A D. Annu Rep Prog Chem Sect A, 2012, 108: 464

[16] Prasek J, Drbohlavova J, Chomoucka J, Hubalek J, Jasek O, Adam V, Kizek R. J Mater Chem, 2011, 21: 15872

[17] Erdemir A, Donnet C. J Phys D, 2006, 39: R311

[18] McKenzie D R. Rep Prog Phys, 1996, 59: 1611

[19] Iakoubovskii K, Baidakova M V, Wouters B H, Stesmans A, Adriaenssens G J, Vul' A Ya, Grobet P J. Diam Relat Mater, 2000, 9: 861

[20] Thaddeus P, McCarthy M C. Spectrochim Acta Part A, 2001, 57: 757

[21] Titirici M-M, White R J, Falco C, Sevilla M. Energy Environ Sci, 2012, 5: 6796

[22] Sevilla M, Fuertes A B. Carbon, 2009, 47: 2281

[23] Sevilla M, Fuertes A B. Chem Eur J, 2009, 15: 4195

[24] Khavryuchenko V D, Khavryuchenko O V. J Phys Chem C, 2013, 117: 7628

[25] Gilbert J B, Kipling J J, McEnaney B, Sherwood J N. Polymer, 1962, 3: 1

[26] Liu Y D, Kumar S. Polym Rev, 2012, 52: 234 